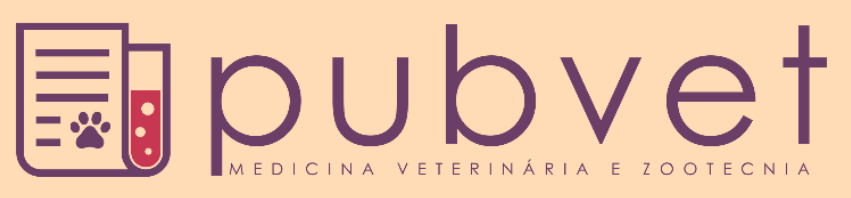

https://doi.org/10.31533/pubvet.v12n10a193.1-5

\title{
Efeitos sociais provocados pelo número de casos de doenças transmitidas pelo mosquito Aedes aegypti no município de Balsas, Maranhão
}

\author{
Ingredy Elkana Andrade Ferreira Medeiros ${ }^{10}$, Geovania Maria da Silva Braga ${ }^{2} \bullet$
}

${ }^{\text {I} P o ́ s ~ g r a d u a n d a ~ e m ~ S a u ́ d e ~ P u ́ b l i c a ~-~ U n i v e r s i d a d e ~ E s t a d u a l ~ d o ~ M a r a n h a ̃ o, ~ I m p e r a t r i z, ~ M a r a n h a ̃ o, ~ B r a s i l . ~ E-m a i l: ~ i n g r e d y ~ e l k a n a @ o u t l o o k . c o m ~}$ ${ }^{2}$ Pós Doutora em Saúde Pública; Profa Adjunto IV - Universidade Estadual da Região Tocantina do Maranhão, Imperatriz, Maranhão, Brasil. E-mail: geovaniacesi.uema@gmail.com *Autor para correspondência

\begin{abstract}
RESUMO. O grau do nível da epidemia é de grande relevância, perante o risco de uma população, que poderá sofrer com várias enfermidades. As doenças transmitidas pelo mosquito Aedes aegypti, por se tratar de doenças que acometem grande parte de pessoas em todo Brasil, causando inúmeros efeitos devastadores, as mesmas requerem a necessidade da construção de uma maior demanda que rege a atenção à saúde dos indivíduos. Este artigo tem por objetivo considerar os efeitos sociais provocados pelo aumento no número de casos de doenças, que são transmitidas através do mosquito Aedes aegypti. Foi efetuado um levantamento de dados dos artigos bibliográficos, quanto ao número de casos das doenças transmitidas pelo vetor em questão em várias regiões. Considerando o alto índice de casos urbano dessas arboviroses causadas pelo referido inseto, no município de Balsas, estado do Maranhão, nos anos de 2016 a 2018, assim como também a incidência das mesmas, em comparação aos outros anos é que se se realizou uma coleta de dados, de acordo com o programa do SINAN NET, observando-se que houve integração de diversas estratégias para controle vetorial, estas compatíveis e disponíveis às características regionais, visando reduzir a proliferação desse inseto e a incidências das arboviroses transmitidas pelo mesmo, no município de Balsas, estado do Maranhão.
\end{abstract}

Palavras-chave: arboviroses, inseto, saúde pública

\section{Social effects caused by the number of cases of disease transmitted by the Aedes aegypti mosquito in the city of Balsas, Brazil}

ABSTRACT. The degree of the level of the epidemic is of great importance, given the risk of a population, which may suffer from various diseases. Since it is diseases that affect a large part of people from all over Brazil, causing numerous devastating effects, they require the need of building a greater demand that governs the attention to health of individuals. This article aims to consider the social effects caused by the increase in the number of cases of diseases that are transmitted through the Aedes aegypti mosquito. Where was made a bibliographic data of articles, the number of cases of vector-borne diseases in question in several regions. Considering the high rate of cases of these urban Arboviruses caused by said insect, in the municipality of Balsas, Maranhão State, in the years 2016 to 2018, as well as the incidence of the same, compared to other years is that if you held a collection of data, in accordance with the program of SINAN NET, observing that there was integration of diverse strategies for vector control, these compatible and available to the regional characteristics, in order to reduce the spread of this insect and the effects of Arboviruses transmitted by the same, in the municipality of Balsas, Maranhão State.

Keywords: Arboviruses, insect, public health. 


\title{
Efectos sociales provocados por el número de casos de enfermedades transmitidas por el mosquito Aedes aegypti en el municipio de Balsas, Brasil
}

\begin{abstract}
RESUMEN. El grado del nivel de la epidemia es de gran relevancia, ante el riesgo de una población, que puede sufrir con varias enfermedades. Dado que por tratarse de enfermedades que afectan a gran parte de personas de todo Brasil, causando innumerables efectos devastadores, las mismas requieren la necesidad de la construcción de una mayor demanda que rige la atención a la salud de los individuos. Este artículo tiene por objetivo considerar los efectos sociales provocados por el aumento en el número de casos de enfermedades que se transmiten a través del mosquito Aedes aegypti. se efectuó un levantamiento de datos en la literatura, sobre el número de casos de enfermedades transmitidas por el vector en cuestión en varias regiones. Considerando el alto índice de casos de estas arbovirosis causadas por dicho insecto, en el municipio de Balsas, estado de Maranhão, en los años 2016 a 2018, así como también la incidencia de las mismas, en comparación a los otros años, se realizó un levantamiento de datos, de acuerdo con el programa del SINAN NET, observándose que hubo integración de diversas estrategias para control vectorial, estas compatibles y disponibles a las características regionales, con el objetivo de reducir la proliferación de ese insecto y las incidencias de las arbovirosis transmitidas por el mismo, en el municipio de Balsas, estado de Maranhão.
\end{abstract}

Palabras clave: arbovirosis, insecto, salud pública

\section{Introdução}

Por se tratar de um mosquito pequeno, de apenas sete milímetros, o Aedes aegypti é capaz de transmitir numerosas doenças diferentes, podendo causar inúmeros efeitos sociais na população, entre elas se destacam: a dengue, o Zika vírus, a Febre amarela, o vírus Mayaro e Chikungunya (Braga \& Valle, 2007a; Braga \& Valle, 2007b). Estas doenças distintas, e com grandes agravos, mesmo tendo sintomatologias semelhantes, não se descarta a relevância e o grau do nível da epidemia. No entanto, a doença que se desenvolve depende do vírus que o mosquito carrega, ou seja, nem todos os mosquitos dessa espécie estão infectados.

O Aedes aegypti é um mosquito procedente do Egito. A disseminação pelo planeta aconteceu da costa leste do continente Africano para as Américas, e depois da costa oeste para a Ásia. O vetor foi descrito cientificamente pela primeira vez em 1762, quando foi denominado Culex aegypti (BRASIL, 2006). O gênero Aedes só foi apresentado em 1818, pois, constatou-se que a espécie aegypti, descrita anos antes, apresenta características morfológicas e biológicas semelhantes às de espécies do gênero Aedes e não às do já conhecido gênero Culex. Desta forma, foi que recebeu o nome Aedes aegypti (Braga \& Valle, 2007a).

A percepção da importância epidemiológica do A. aegypti ocorreu em 1881, quando ele foi confirmado como transmissor da febre amarela, por Finlay (1940). Em 1906, Brancroft divulgou as primeiras evidências de que o mosquito também era o vetor da dengue, fato posteriormente confirmado por Agramonte, em 1906 e por Simmons, em 1931 (Martinez, 1990; WHO, 1997). Dessa forma é importante sempre abordar a saúde coletiva, tendo em vista o enfoque epidêmico onde requer a compreensão do processo evolutivo no âmbito social, cultural, político e econômico que influenciam na expansão ou retração de uma epidemia. Da mesma forma, a dimensão geográfica (climática, socioambiental e urbana) na qual se desenvolve o processo saúdedoença da população.

Faz-se referência à dengue no Brasil desde 1846 (Gonçalves Neto et al., 2006). Entretanto, somente na metade do século XX, a partir de 1986, a dengue adquiriu importância epidemiológica, quando apareceu como epidemia no estado do Rio de Janeiro e a circulação do sorotipo (DEN-1) que atingiu a região Nordeste. Registros de epidemias são referenciados em Niterói (RJ) desde 1923, sem confirmação laboratorial.

A primeira epidemia no Brasil com confirmação laboratorial é datada de 1982 no estado de Roraima. Em 1986, manifesta-se novamente no estado do Rio de Janeiro, onde ocorreu uma grande epidemia, juntamente com os estados de Alagoas e Ceará (Gonçalves Neto et al., 2006). Destaca-se entre as enfermidades reemergentes, sendo considerada a mais importante das doenças virais transmitidas por artrópodes no mundo (Tauil, 2014). A doença apresenta um 
padrão sazonal, com maior incidência de casos nos primeiros cinco meses do ano, período mais quente e úmido, típico dos climas tropicais. Dessa forma, a dengue se tornou endêmica no Brasil, intercalando-se epidemias, geralmente associadas à introdução de novos sorotipos, em áreas anteriormente indenes (FNS, 2001).

O Zika Vírus é um arbovírus do gênero Flavivírus, pertence à família Flaviviridae (ehler et al., 2014). Inicialmente foram subestimadas por serem transmitidas pelo mosquito $\mathrm{e}$ se assemelharem aos sintomas da dengue (BRASIL, 2015a). Um artigo publicado na revista The Lancet no início de 2016 mostrou que, quando a doença se trata de gestantes, os efeitos podem recair sobre o feto provocando um aumento do número de bebês com microcefalia (Vasconcelos, 2015). Conforme Cugola et al. (2016), apesar de ser uma infecção viral considerada leve e, na maioria dos casos, o Zika pode acometer o sistema nervoso central, sendo associada a síndrome de Guillian-Barré (Vedanaryanan \& Chaudhry, 2000). Em uma epidemia ocorrida na Micronésia foram registrados 40 casos da síndrome de Guillian-Barré. Segundo Zorzetto (2008) pesquisas apontam que o zika brasileiro é mais agressivo que o vírus africano. Vírus chikungunya $(C H I K V)$ é um alfavírus africano que pertence ao mesmo genótipo do vírus Mayaro (MAYV) (Nunes, 2005). O significado de chikungunya vem da língua africana makonde que significa "aquele que é contorcido", devido à forte dor causada nas articulações (Tauil, 2014).

No Brasil, a transmissão da chikungunya foi detectada em setembro de 2014, na cidade de Oiapoque, estado do Amapá (Donalisio \& Freitas, 2015). A infecção desta doença muita das vezes se recorda a causada pelo vírus da Zika e da dengue, motivo pela qual o diagnóstico correto só é possível por meio de testes moleculares (PCR) e imunológicos (Severo et al., 2016). O MAYV tem sido responsável por surtos de doença febril aguda e síndrome de artralgia na Região Amazônica e no Planalto Central do Brasil, bem como em outros países da América do Sul (Peru, Bolívia e Venezuela) (BRASIL, 2015b). Casos humanos são esporádicos e ocorrem principalmente em pessoas egressas de florestas tropicais úmidas.

A febre do Mayaro é uma doença infecciosa febril aguda, causada por um arbovírus (vírus transmitido por artrópodes), que pode causar uma doença de curso benigno semelhante a dengue. Normalmente, após uma ou duas semanas o paciente se recupera completamente (Wermelinger \& Carvalho, 2016). A transmissão pelas vias aéreas, como meio de disseminação viral, pode ter sido a fonte de uma infecção acidental, em laboratório. Portanto, pode ser potencialmente uma via de transmissão natural, facilitando assim a disseminação do vírus ocasionando surtos (Braga \& Valle, 2007a). Os sintomas da febre Mayaro são muito parecidos com os da dengue e por isso pode ser facilmente confundida com dengue ou com chikungunya, nestes casos, os testes sorológicos são os mais indicados (BRASIL, 2015b).

A partir de 1996, o Ministério da Saúde do Brasil colocou em prática o Plano de Erradicação, PEAa, pelo Programa de controle do Ae. aegypti. O mesmo preconizava a atuação multi-setorial e os avanços das ações de controle e prevenção das arboviroses no Brasil. Isso decorre do fato que ainda não tem vacina disponível e medicamentos eficazes contra estas arboviroses. Desta forma, as recomendações preconizadas pelo Ministério da Saúde se restringem, principalmente, a ações de combate aos vetores intra-domiciliares, eliminando os possíveis criadouros.

\section{Efeitos sociais provocados pelo número de casos de doenças transmitidas pelo mosquito Aedes aegypti no município de Balsas, Maranhão}

Considerando o alto índice de casos urbanos de arboviroses transmitidas pelo mosquito Aedes aegypti e a incidência das mesmas, em comparação aos outros anos, no município de Balsas, estado do Maranhão, nos anos de 2016 a 2018, foi realizada uma coleta de dados, de acordo com o programa do SINAN NET, neste município.

No município de Balsas Maranhão, observamse inúmeros casos suspeitos das arboviroses, entre estas Dengue, Febre Chikungunya e Zika Vírus. De acordo com o programa do SINAN NET, nos anos de 2016 a 2018 foram registrados 8.214 casos suspeitos de arboviroses (Tabela 1). Os casos notificados nos dados epidemiológicos deste município, mostram a necessidade de avanços no controle dessas infestações, sabendo se que há uma grande proporção desses casos no período de chuvas.

Visto que, por se tratar de enfermidades que acometem grande parte de pessoas em todo o território nacional, causando inúmeros efeitos devastadores, as mesmas requerem a necessidade 
da construção de uma maior demanda que rege a atenção à saúde dos indivíduos. Sendo assim, por meio desta pesquisa buscou-se ampliar o conhecimento do processo dos efeitos destas doenças associadas a fatores sociais, observando para a prevenção das mesmas, com intuito de minimizar os impactos na segurança pessoal.

Tabela 1. Casos notificados dos dados epidemiológicos suspeitos das arboviroses no município de Balsas, estado do Maranhão, 2018.

\begin{tabular}{lccc}
\hline Ano & Dengue & $\begin{array}{c}\text { Febre } \\
\text { Chikungunya }\end{array}$ & Zika Vírus \\
\hline 2016 & 2.101 & 1.584 & 14 \\
2017 & 1.910 & 2.506 & 63 \\
2018 & 21 & 12 & 03 \\
\hline
\end{tabular}

CS = Casos Suspeitos. Fonte: SINAN NET (2018).

Tendo como base de intervenção, os profissionais da saúde e todo departamento epidemiológico, seguindo todo o protocolo de prevenção contra o inseto vetor. A integração de diferentes estratégias de controle vetorial compatíveis e eficazes, sempre será o meio mais adequado quando se trata dos protocolos, programas de controle, prevenção e tratamento dessas doenças, considerando principalmente as tecnologias disponíveis e as características regionais, estas por parecer ser um método viável para tentar reduzir a infestação dos mosquitos e a incidência das arboviroses transmitidas por eles.

Estes dados são de grande relevância levandose em consideração que, ainda não se tem vacina disponível para todas as arboviroses, e nem medicamentos eficazes contra estas enfermidades. Assim sendo, as recomendações preconizadas pelo Ministério da Saúde que se restringem, principalmente às ações de pugna aos vetores intradomiciliares, eliminando os possíveis criadouros. A situação de outras regiões onde o índice destas enfermidades é bastante elevado, não é diferente do município de Balsas, no estado do Maranhão, onde dá para perceber-se que existe um controle por meio dos agentes de endemias, que orientam a comunidade pelas visitas domiciliares, aplicação de produtos para domínio e assim conscientizando sobre os meios de prevenção, sintomas da doença, influência dos impactos sociais.

\section{Considerações finais}

Os efeitos sociais provocados pelo aumento do número de casos de doenças transmitidas pelo mosquito Aedes aegypti de forma abrangente, tendo em vista o enfoque epidêmico, onde requer a compreensão do processo evolutivo, no âmbito social, cultural, político e econômico, bem como da dimensão geográfica, como climática, socioambiental e urbana, na qual se desenvolve o processo saúde-doença da população é bastante expressivo no município de Balsas, estado do Maranhão.

\section{Agradecimentos}

Ao Curso de Pós-graduação em Saúde Pública da Universidade Estadual do Maranhão - UEMA.

\section{Referências}

Braga, I. A., \& Valle, D. 2007a. Aedes aegypti: histórico do controle no Brasil. Epidemiologia e Serviços de Saúde, 16(2), 113-118.

Braga, I. A., \& Valle, D. 2007b. Aedes aegypti: inseticidas, mecanismos de ação e resistência. Epidemiologia e Serviços de Saúde, 16(4), 279-293.

BRASIL. 2006. Doenças infecciosas e parasitárias: guia de bolso: Departamento de Vigilância Epidemiológica. Secretaria de Vigilância em Saúde. Ministério da Saúde.

BRASIL. 2015a. Boletim Eipidemiológico (Vol. 24). Brasília, Brasil: Departamento de Vigilância Epidemiológica. Secretaria de Vigilância em Saúde. Ministério da Saúde.

BRASIL. 2015b. Febre de Chikungunya: Manejo clínico (Vol. 28). Brasília, Brasil: Departamento de Vigilância Epidemiológica. Secretaria de Vigilância em Saúde. Ministério da Saúde.

Cugola, F. R., Fernandes, I. R., Russo, F. B., Freitas, B. C., Dias, J. L. M., Guimarães, K. P. \& Romero, S. 2016. The Brazilian Zika virus strain causes birth defects in experimental models. Nature, 534(7606), 267.

Donalisio, M. R., \& Freitas, A. R. R. 2015. Chikungunya in Brazil: an emerging challenge. Revista Brasileira de Epidemiologia, 18(1), 283-285.

Finlay, C. E. 1940. Carlos J. Finlay. Oxfod: Oxford University Press New York.

FNS. 2001. Plano de intensificação das ações de controle da dengue. Brasilia, Brasil: Ministério da Saúde.

Gonçalves Neto, V. S., Monteiro, S. G., Gonçalves, A. G., \& Rebêlo, J. M. M. 2006. Conhecimentos e atitudes da população sobre 
dengue no Município de São Luís, Maranhão, Brasil, 2004. Cadernos de Saúde Pública, 22(10), 2191-2200.

Martinez E. M. 1990. Dengue hemorrágico en niños. Habana: Ed. José Marti.

Nunes, M. R. T. 2005. Isolamento de vírus Chikungunya, Sudeste do Brasil. Doenças Infecciosas Emergentes, 11, 1610-1613.

Oehler, E., Watrin, L., Larre, P., Leparc-Goffart, I., Lastere, S., Valour, F., Ghawche, F. 2014. Zika virus infection complicated by GuillainBarre syndrome-case report, French Polynesia, December 2013. Eurosurveillance, 19(9), 7-9.

Severo, C. S., Martinez, F. D., Rauber, J. G., Benacchio, A., \& Costa, E. B. B. 2016. Sistema Aedes Gamificado. Instituto Federal do Paraná, 1, 1-4.

SINANWEB - Sinan Net - Ministério da Saúde. Disponivel: www.portalsinan.saude.gov.br/ sinan-net/43-institucional. Acesso: 28.06.18.

Tauil, P. L. 2014. Condições para a transmissão da febre do vírus chikungunya. Epidemiologia e Serviços de Saúde, 23(4), 773-774.

Vasconcelos, P. F. C. 2015. Doença pelo vírus Zika: um novo problema emergente nas
Américas? Revista Pan-Amazônica de Saúde, 6(2), 9-10.

Vedanaryanan, V. V., \& Chaudhry, V. 2000. Guillian Barré Syndrome-Recent advances. The Indian Journal of Pediatrics, 67(9), 635646.

Wermelinger, E. D., \& Carvalho, R. W. 2016. Métodos e procedimentos usados no controle do Aedes aegypti na bem-sucedida campanha de profilaxia da febre amarela de 1928 e 1929 no Rio de Janeiro. Epidemiologia e Serviços de Saúde, 25(4), 837-844.

World Health Organization - WHO. 1997. Executive committee of the directing council the regional committee Pan American World Health 120th Meeting CE120/21. Geneva.

Zorzetto, R. 2008. Uma doença anunciada. Pesquisa Fapesp, São Paulo, 151, 1-10.

Recebido: 5 julho. 2018 .

Aprovado: 27 agosto. 2018

Publicado: 23 outubro 2018

Licenciamento: Este artigo é publicado na modalidade Acesso Aberto sob a licença Creative Commons Atribuição 4.0 (CC-BY 4.0), a qual permite uso irrestrito, distribuição, reprodução em qualquer meio, desde que o autor e a fonte sejam devidamente creditados. 